\title{
Frustration effect following changed S-R temporal relations
}

H. D. KIMMEL, OHIO UNIVERSITY NANCY HOOTER MCGINNIS, UNIVERSITY OF FLORIDA

Twenty-four rat $S$ s received training to run down a $6 \mathrm{ft}$. runway for food reward with either a 2 or $4 \mathrm{sec}$. interval between a discrete click and being dropped onto the runway. Half the $S s$ in each group were then shifted to the other time interval while the remaining half of each group continued with its original interval. It was found that increasing the time interval between the click and placement in the runway led to an increase in speed of running which grew from the first to the second day of testing. Reducing the interval following the click had the effect of slowing the shifted animals, but this effect vanished on the second test day. It was concluded that the increase in the time interval following the click produced a frustration effect (a motivational effect) while the decrease in the time interval led to generalization decrement (an associative effect), followed by new learning at the new interval.

The effects of enforced changes in temporal S-R relations in an instrumental reward situation were studied in this experiment, the changes being either an increase or a decrease in time between events immediately prior to the overt instrumental behavior. After being placed in the start apparatus, the $S$ received an auditory stimulus (a click). Either 2 or 4 sec. later, the floor of the start apparatus dropped from below the $S$, placing it at the beginning of a $6 \mathrm{ft}$. runway. Traversing the runway led to food reward. It was hypothesized that, after such training, an increase in the time between the click and being dropped. onto the runway would result in an increase in the speed of the instrumental running response, as a consequence of frustration produced by delay of "reinforcement," while a decrease in time between the click and being dropped would result in attenuation of the speed of the running response, as a consequence of generalization decrement occasioned by the requirement that the response be performed before the appropriate $r_{G^{-s}}$ events had occurred. The frustrationproduced acceleration was thought of as a motivational effect while the generalization decrement-produced attenuation was thought of as an associative effect. Method

Twenty-four male albino rats, 150-180 days old, served as Ss. A detailed description of the runway has been presented previously (Brown, Martin, \& Morrow, 1964). It consisted of a start box divided into upper and lower compartments by a trap-door floor 7 in. above the bottom floor, a $6 \mathrm{ft}$. runway, and a goal box. All inside walls were painted white except the goal box, which was flat black. The click was produced by E's snapping a metal rod against the wall of the start box. This also started a timer which operated the trap-door floor either 2 or 4 sec. later. S's interruption of a photoelectric beam at the entrance to the runway started a Klockounter which stopped when a second beam was crossed at the entrance to the goal box. This "running time" measure was obtained on each training and testing trial.

The Ss were first given 10 days of ad lib feeding and handling. A measure of adlib weight was obtained on the last two days of this period. Ss were then starved (with access to water) until they reached $80 \%$ of ad lib weight. Each S received only enough food each day thereafter to maintain the $80 \%$ weight. Water was available in the home cages. On the first day after the weight criterion was reached the $S$ was placed in the goal box where it found one Noyes pellet. Ten trials of this type were given. On the second day the Straversed $2 \mathrm{ft}$. of the runway to get to the goal box for one pellet. Five such trials were given. On the third and fourth days 10 trials in a $4 \mathrm{ft}$. runway were given. On the fifth day 10 trials were run in the $6 \mathrm{ft}$. runway, without using the start box dropping device. On these trials the $\mathrm{S}$ was placed directly under the trap-door floor to begin each trial.

The Ss were then randomly divided into two groups for 10 training trials using the start box trap-door floor and the click. Ss in the $2 \mathrm{sec}$. group were placed into the upper part of the start box and, after 3-5 sec., the click was presented. Two sec. later the floor dropped and the $S$ ran down the runway to the goal box for one pellet. The Ss in the $4 \mathrm{sec}$. group were treated identically except that the interval between the click and the operation of the trap-door was $4 \mathrm{sec}$. For the next two days the Ss of each training group were randomly separated into testing subgroups, half of the animals continuing to have the same interval between the click and the drop while the other half were shifted to the other time interval. Ten trials were given on each testing day.

\section{Results}

Figure 1 shows the mean log running time in 2-trial blocks for the last 10 training trials and the 10 trials on each test day. Training under the 2 or $4 \mathrm{sec}$. condition appeared to produce little difference between the groups and both groups tended to run faster at the end of training than at the start.

The second panel of Fig. 1 (1st test day) shows that the 2 sec. group that was shifted to 4 sec. tended to run faster than the group that was continued at the $2 \mathrm{sec}$. interval. This difference was more pronounced on the second test day, as is shown in the third panel. The 4 sec. group that was shifted to $2 \mathrm{sec}$. ran considerably 


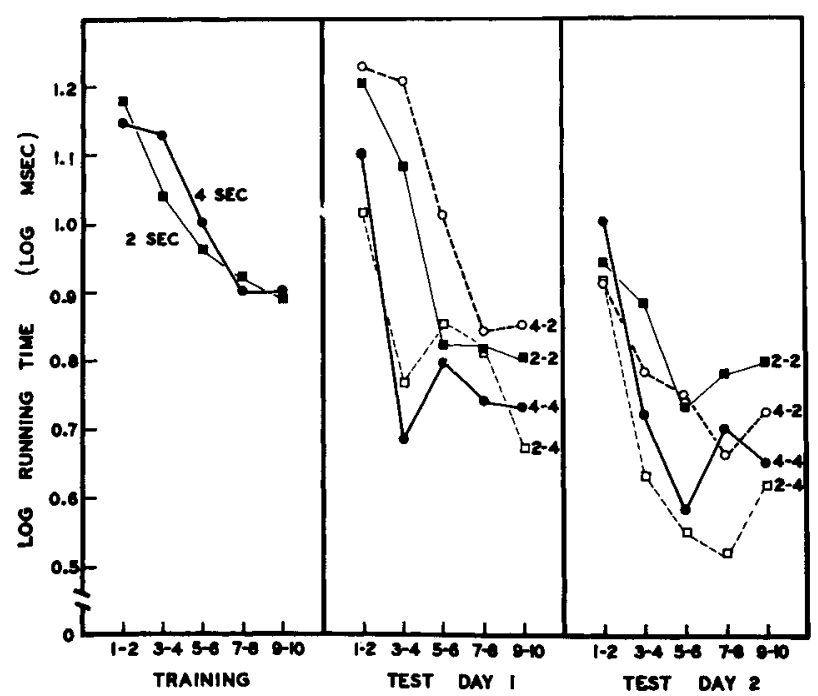

Fig. 1. Mean transformed running times on last day of training and two days of testing, in two-trial blocks.

slower than the group that remained at 4 sec. This difference tended to diminish on the second test day.

Analysis of variance of these data revealed that the triple interaction of Training interval, Testing interval, and Days of testing was significant, $\mathrm{F}=4.75, \mathrm{df}=1 / 20$, EMS $=0.17$. The group shifted from the $4 \mathrm{sec}$. interval to the $2 \mathrm{sec}$. interval ran decidedly slower than the unshifted 4 sec. group on the first test day but recovered almost completely on the second day. The difference between the two $4 \mathrm{sec}$. groups was significant on the first test day, $t=2.45, d f=20$, but not on the second day or on both days combined. Comparison of the two $2 \mathrm{sec}$. groups indicated that the shifted group ran somewhat faster on the first test day, but not significantly, $t=1.31$, $\mathrm{df}=20$, and that the superiority of the shifted group was greater on the second test day, but again not quite significantly, $t=1.93, \mathrm{df}=20$. On both days combined the group shifted from 2 to $4 \mathrm{sec}$. ran significantly faster than the unshifted 2 sec. group, $t=2.74, d f=20$.

\section{Discussion}

The results indicated that when an animal is trained to perform an instrumental response to receive reward, delaying the temporal sequence of events justpreceding the trained behavior has the effect of increasing the speed with which the eventual response is performed. It is significant that the overt, measurable response was not, itself, interrupted, but that the animal was merely forced to wait longer in the startbox before the response could be performed. What must have been interrupted was a hypothetical chain of $r_{G^{-s}}{ }_{G}$ events that are assumed to have been the basis of the S's "timing" ability. The result is quite analogous to the FE produced by interrupting the overt instrumental response (Amsel, 1958). The fact that the effect increased from the first to the second test day is taken as support for the conclusion that it was motivational rather than associative. Reducing the time between prior events, as was done in the group that was shifted from $4 \mathrm{sec}$. to $2 \mathrm{sec}$, produced not enhancement but impairment of the running behavior. Furthermore, this effect was almost gone by the second day of testing, which we interpret to mean that the Ss in this group were able quickly to re-learn the task at the shortened interval. Shortening the interval required that the animal run "before it was ready," a condition conducive to generalization decrement.

\section{References}

Amsel, Abram. The role of frustrative nonreward in noncontinuous reward situations. Psychol. Bull., 1958, 55, 102-106.

Brown, J. S., Martin, R. C., \& Morrow, M. C. Self-punitive behavior in the rat: Facilitative effects of punishment on resistance to extinction. J. comp. physiol. Psychol, 1964, 57, 127-133. 